\title{
Mixing Times of Plane Random Rhombus Tilings
}

\author{
Nicolas Destainville \\ Laboratoire de Physique Quantique, UMR-CNRS 5626, \\ Université Paul Sabatier, 118 route de Narbonne, 31062 Cedex 5, Toulouse, France
}

\begin{abstract}
We address the question of single flip discrete dynamics in sets of two-dimensional random rhombus tilings with fixed polygonal boundaries. Single flips are local rearrangements of tiles which enable to sample the configuration sets of tilings via Markov chains. We determine the convergence rates of these dynamical processes towards the statistical equilibrium distributions and we demonstrate that the dynamics are rapidly mixing: the ergodic times are polynomial in the number of tiles up to logarithmic corrections. We use an inherent symmetry of tiling sets which enables to decompose them into smaller subsets where a technique from probability theory, the so-called coupling technique, can be applied. We also point out an interesting occurrence in this work of extreme-value statistics, namely Gumbel distributions.
\end{abstract}

Keywords: Random tilings, Discrete dynamical systems, Markovian processes, Quasicrystals.

\section{Introduction}

After the discovery of quasicrystals about 15 years ago [1], Penrose tilings [2] and more generally quasiperiodic tilings as well as their randomized counterparts - namely random rhombus tilings - rapidly became a popular topic for a large community of researchers. Indeed, they appeared to be suitable paradigmatic models for quasicrystalline alloys [3, 4]. However, it rapidly became clear that characterizing and establishing the properties of this new class of models in solid state and statistical physics would be a formidable and long-term task. Simultaneously, these systems also became an active field of investigation for computer scientists and mathematicians due to the richness of their properties (see [5] or [6] for examples).

An example of random tiling is displayed in figure 1. It belongs to the class of two-dimensional tilings with octagonal (or 8-fold) symmetry. Beyond this example, plane tilings with larger symmetries including Penrose tilings [2] and space tilings with icosahedral symmetries were proposed to model every kind of real quasicrystal. Indeed, the complex microscopic structures of the highest quality alloys turn out to be faithfully described by these tilings. Note however that the origin of the stability of quasicrystals is still debated. Physical explanations ranging from an electronic stabilization in perfect quasiperiodic structures to an entropic stabilization allowed by the large amount of microscopic configurations in a random tiling ensemble have been put forward. Our purpose is not to discuss the relative merits of these approaches but to analyze the combinatorial problematic associated with the random tiling model [7]. 


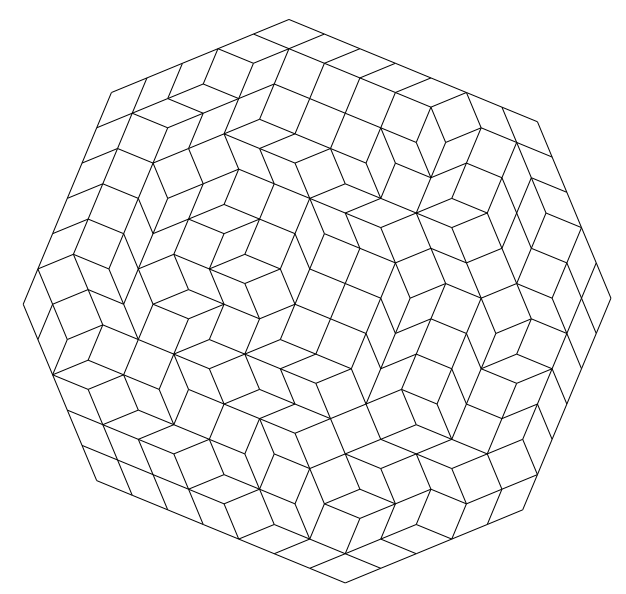

Fig. 1: Example of randomly sampled fixed boundary tiling. It fills a centrally symmetric octagonal region of sides $5,6,5$ and 7 .

The present paper is devoted to dynamical properties of random rhombus tilings in terms of local dynamical rules, the so-called phason-flips, which consists of local rearrangement of tiles. It is the activation of these degrees of freedom which gives access to a large number of configurations in the random tiling model. These dynamics are of interest for several reasons.

On the one hand, it is more and more clear that phason-flips exist in real quasi-crystals (see [8]) and can be modeled in a first approximation by tile-flips; they are local rearrangements of atoms and are therefore a new source of atomic mobility, as compared to usual crystalline materials. In particular, these atomic moves could carry their own contribution to self-diffusion in quasicrystalline materials, as was first pointed out in reference [9], even if the efficiency of such processes in real alloys remains controversial [10]. Moreover, they are certainly involved in some specific mechanical properties of quasicrystals, such as plasticity via dislocation mobility [11]. The two processes are related since long-range diffusion is necessary to allow dislocation formation [11]. Therefore a complete understanding of flip dynamics is essential in quasicrystal physics.

On the other hand, as it became clear that the characterization of statistical properties of random tiling sets would be a long process, a lot of numerical work has been carried out, a part of which was based on Monte Carlo techniques which rely on a faithful sampling of the tiling sets (see [12, 13, 14] for examples). So far, no systematic study of the relaxation times between two independent numerical measures has been accomplished, whereas it is an essential ingredient for a suitable control of error bars. However, there exist exact results in the simplest case of random rhombus tilings with hexagonal symmetry [15, 16, 17] and several estimates of relaxation times in larger symmetries, either numerical or in the approximate frame of Langevin dynamics [12, 13, 18]. Note that these approximate results miss logarithmic corrections provided by an exact treatment.

In this paper we examine, by probability theory techniques, mixing times of flip dynamics, also called correlation or ergodic times, which characterize how many flips the system needs to (nearly) forget its initial configuration and to reach any configuration with uniform probability, in other words at which rate the system converges towards its stationary distribution. Indeed, we suppose here that all tilings have the 
same probability; in physical terms we work at infinite temperature.

As compared to the hexagonal symmetry case, the main complication in the analysis of mixing times in sets of rhombus tilings with 8-fold or larger symmetries seems to come from the fact that there is no convenient way to define underlying lattices in these tilings. Our method relies on the general idea that these sets can be disconnected (or decomposed) in smaller subsets, in each of which all tilings can be mapped onto families of paths ("routings") running on an underlying rhombus tiling with smaller symmetry [19]. On each subset, which is called a "fiber", we will establish that the flip dynamics is rapidly mixing. Then the difficulty will be to reconnect the different fibers and to reconstruct the initial dynamics, in order to show that it is in turn rapidly mixing.

Before going on, we mention that this paper is restricted to tilings with fixed polygonal boundaries, for the sake of technical simplicity [20].

The first section is devoted to preliminaries (state of the art, definitions, known techniques and results). In particular, we present in this section the coupling method to estimate mixing times and the "disconnection" process of the tiling set. The second section contains the main results of the paper, in the specialized case of octagonal tilings: mixing times on fibers and the "reconnection" process. Beyond the octagonal case, the last section is devoted to the generalization of our results to the larger class of plane rhombus tilings with any $2 D$-fold symmetry. Appendices are devoted to technical proofs or developments, which need not be exposed in the body of the paper.

This paper follows and completes a shorter version which was dedicated to octagonal tilings only [21].

\section{Preliminaries}

\subsection{Definitions and state of the art}

Random rhombus tilings of plane (and of space) are made of rhombi of unitary side length. They are classified according to their global symmetry groups [7]. The simplest class of hexagonal tilings - made of $60^{\circ}$ rhombi with 3 possible orientations - has been widely explored. Indeed, these tilings are commonly mapped onto assemblies of random walkers on square lattices, or "routings" (see [22] or [16] for instance), which highly facilitates their study. Tilings with octagonal symmetry are made of 6 different tiles: two squares differently oriented and four $45^{\circ}$ rhombi (see figures 1 and 2). Beyond these two cases, one can define tilings with higher symmetries (e.g. Penrose tilings [2]) or of higher dimensions [7]. For sake of technical simplicity, we focus on tilings filling a centrally symmetric polygonal region with integral side lengths (figure 1; see also reference [20]). For a $2 D$-fold symmetry, this polygon is a $2 D$-gon. As it is usual in the field of random tilings, we are interested in the large size limit where the polygon becomes infinitely large, while keeping a fixed shape: its $i$-th side is equal to $x_{i} k$, where $x_{i}$ remains fixed while $k$ goes to infinity (see also reference [20]). However, most of our statements will concern the diagonal case $x_{1}=x_{2}=\ldots=x_{D}=1$ for the sake of simplicity but can easily be extended to non-diagonal cases.

As far as dynamics is concerned, these tiling sets are endowed with rearrangements of tiles. In this paper, we will focus on local 3-tile flips, or elementary flips: as figure 2 illustrates it, whenever 3 rhombic tiles fill an hexagon in a tiling, they can be "rotated" in that hexagon, thus generating a new tiling.

The set of all the tilings of such a region together with the above discrete dynamical rule define a discrete time Markov chain [23, 24]: at each step, a vertex of the tiling is uniformly chosen at random and if this vertex is surrounded by 3 tiles in flippable configuration, then we flip it. Starting from an initial tiling, this process can reach certain configurations with certain probabilities. Since the sets of plane tilings are 


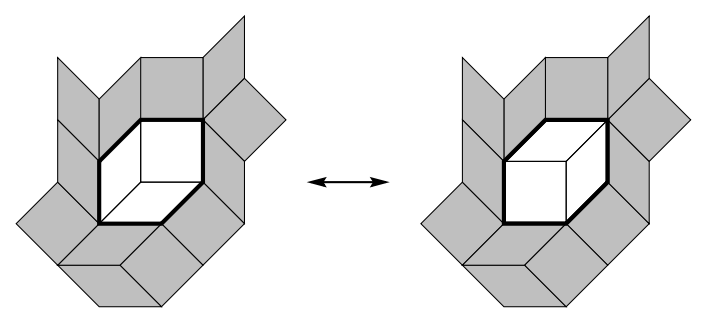

Fig. 2: An example of elementary flip, involving 3 (white) tiles, in a patch of octagonal random tiling.

connected via elementary flips [25, 19], this process can reach any tiling. Moreover, it converges toward the uniform stationary (or equilibrium) distribution, since it satisfies the detailed balance condition (in probability theory, one says equivalently that the Markov chain is reversible [24]). All the difficulty is to characterize the convergence rates: how many flips are necessary to get close to this stationary distribution? So far they have only been calculated for hexagonal tilings [15, 17]: the characteristic times behave like the square of the system size, up to logarithmic corrections. Note that in this specific case, this result still holds for more general, non-local tile flips, the so-called tower moves [15, 17]. Before going on, we need to define precisely such characteristic times, and therefore firstly how to measure the distance from the probability distribution at any time to equilibrium.

Generally speaking, let us consider a Markov chain on a finite configuration space $L$, which converges toward a stationary distribution $\pi$ on $L$. Let $x_{0}$ denote an initial configuration and $P\left(x, t \mid x_{0}, 0\right)$ be the probability that the process has reached the configuration $x$ after $t$ steps. Then the variation distance

$$
\Delta\left(t, x_{0}\right)=\frac{1}{2} \sum_{x \in L}\left|P\left(x, t \mid x_{0}, 0\right)-\pi(x)\right| \leq 1
$$

measures the distance between this distribution $P$ and the stationary one $\pi$ [26]. Given $\varepsilon>0$ we define

$$
\tau\left(x_{0}, \varepsilon\right)=\inf _{t_{0}}\left\{t_{0} / \forall t \geq t_{0}, \Delta\left(t, x_{0}\right) \leq \varepsilon\right\}
$$

and finally the ergodic or mixing time

$$
\tau(\varepsilon)=\sup _{x_{0}} \tau\left(x_{0}, \varepsilon\right)
$$

Hence, whatever the initial configuration, after $\tau(\varepsilon)$ steps, one is sure to be within distance $\varepsilon$ of the stationary distribution [26].

\subsection{Coupling techniques for estimation of mixing times}

The coupling technique has been widely explored in the two last decades (see reference [26] for an introduction) and has been successfully applied to the estimation of mixing times of several Markov chains, in particular to the case of hexagonal tilings $[16,17]$. It relies on the surprising idea that it might be simpler to follow the dynamics of couples of configurations instead of a single one, provided this dynamics satisfies some not very constraining conditions. Since this technique might be new to the reader, we shall take the time to introduce it. 
A coupling is a Markov chain on $L \times L$; couples of configurations are updated simultaneously and are strongly correlated, but each configuration, viewed in isolation, performs transitions of the original Markov chain under interest. Moreover, the coupled process is constructed so that when the two configurations happen to be identical, then they follow the same evolution and remain identical forever (coalescence property). Then the central idea of the technique is that the average time the two configurations need to couple (or to coalesce) provides a good upper bound on the original mixing time $\tau(\varepsilon)$. More precisely, given an initial couple $\left(x_{0}, y_{0}\right)$ at time $t=0$, define the coalescence time as

$$
T\left(x_{0}, y_{0}\right)=\inf \left\{t / x(t)=y(t) \mid x(0)=x_{0}, y(0)=y_{0}\right\}
$$

and the coupling time as

$$
T=\sup _{\left(x_{0}, y_{0}\right) \in L \times L}<T\left(x_{0}, y_{0}\right)>,
$$

where the last mean is taken over realizations of the coupled Markovian process. The following result links the mixing time of the original Markov chain to this coupling time $[26,16]$ :

$$
\tau(\varepsilon) \leq T e \ln (1 / \varepsilon)+1 \simeq T e \ln (1 / \varepsilon) .
$$

All the difficulty consists in exhibiting Markovian processes where this method can be technically implemented. In particular, the coalescence property is an essential ingredient of the coupling technique.

If the configuration set $L$ can be endowed with a partial order relation $\preceq$ with unique minimum and maximum elements (denoted by $\hat{0}$ and $\hat{1}$ ), the implementation of the technique is highly facilitated, provided the coupled dynamics is monotonous. By monotonous, we mean that if $x(t) \preceq y(t)$, then $x(t+1) \preceq y(t+1)$. In this case, let $\left(x_{0}, y_{0}\right)$ be any initial configuration: $\hat{0} \preceq x_{0} \preceq y_{0} \preceq \hat{1}$, and after any number of steps, the four configurations remain in the same order. Therefore when the iterates of $\hat{0}$ and $\hat{1}$ have coalesced, one is sure that the iterates of $x_{0}$ and $y_{0}$ also have. As a consequence, $T\left(x_{0}, y_{0}\right) \leq T(\hat{0}, \hat{1})$ and the coupling time satisfies $T=<T(\hat{0}, \hat{1})\rangle$.

\subsection{De Bruijn fibration of the configuration space}

A convenient representation of random rhombus tilings was introduced by de Bruijn [27, 28]. It consists of following in a random tiling lines of tiles made of adjacent tiles sharing and edge with a given orientation. Figure 3 (left) displays such de Bruijn lines in an octagonal tiling. The set of lines associated with an orientation is called a de Bruijn family. In a $2 D$-fold tiling, there are $D$ orientations of edges and therefore $D$ de Bruijn families. In this representation, a tile corresponds to the intersection of two de Bruijn lines.

When removing a de Bruijn family from a $2 D$-fold tiling, one gets a $2(D-1)$-fold tiling. Conversely, this remark enables one to propose a convenient iterative algorithm for generating random tilings (see [14, 19]): paths are chosen on a 2(D-1)-fold tiling. They are represented by dark lines in figure 3 (right). They go from left to right and do not intersect (but they can have contacts; see also figure 4). When they are "opened" following a new edge orientation, they generate de Bruijn lines of a new $D$-th family. There is a one-to-one correspondence between $D$-tilings and families of non-intersecting paths on a $(D-1)$ tiling. The latter is called the base tiling. In the following, this idea will be adapted to fixed-boundary tilings in order to study their dynamical properties. At this point, note that a tiling flip involving tiles of the $D$-th family become a line flip in this representation, as illustrated in the figure.

The combinatorial structure of plane rhombic tiling sets has been studied in detail [19, 6]. Unfortunately, even if these sets have unique maximum and minimum elements, which could simplify greatly the 

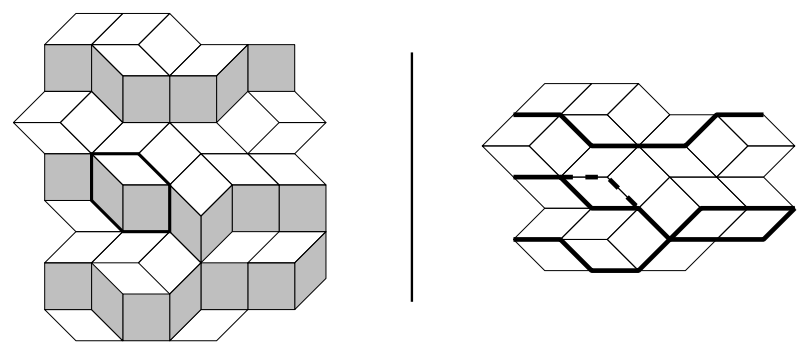

Fig. 3: Left: a de Bruijn family (among 4) in a patch of octagonal tiling; Right: iterative process for the construction of octagonal tiling, the left-hand side tiling can be seen as a family of non-intersecting oriented paths on a hexagonal base tiling. The tiling flip inside the black hexagon (left) would be translated into a line flip (right): the line jumps from one side of a tile to the opposite side (broken line). The same viewpoint can be applied to the polygonal boundary case.

implementation of a coupling technique, defining couplings on the whole configuration spaces seems to be an infeasible task. Consequently we shall instead use the paths-on-tiling point of view defined above to decompose the configuration space into smaller subsets on which couplings can easily be defined. The main difficulty will be then to connect those subsets in order to re-construct the complete dynamics.

We consider each set of tilings which have the same base hexagonal tiling as a subset $J_{a}$ of the whole configuration space $L ; L$ is the disjoint union of the $J_{a}$ 's. In reference [19], these subsets are improperly called "fibers"; nevertheless, we shall go on using this terminology which will prove to be illuminating in the following. In this point of view, the only possible flips in $J_{a}$ are those which involve a given family of de Bruijn lines, which is arbitrarily chosen. Since there are four families of lines, there are four ways to construct such a fibration.

Now, let $\mathcal{M}$ denote the transition matrix associated with the complete Markov chain on the whole set on octagonal tilings: given two configurations $x$ and $y$, the matrix coefficient $\mathcal{M}(x, y)$ is equal to the transition probability $P(x, t+1 \mid y, t)$. In the same way, we define the transition matrices $\mathcal{M}_{i}$ associated with the Markov chains where only flips involving the $i$-th de Bruijn family are allowed. Note first that if $M_{i}^{(a)}$ is the transition matrix on the fiber $J_{a}$ of the fibration $i$-also denoted by $J_{i, a}$-, then $\mathcal{M}_{i}$ is block-diagonal, where each block corresponds with a fiber:

$$
\mathcal{M}_{i}=M_{i}^{(1)} \oplus \ldots \oplus M_{i}^{\left(N_{i}\right)}
$$

where $N_{i}$ is the number of fibers $J_{i, a}$ in the fibration $i$.

As compared to $\mathcal{M}, \mathcal{M}_{i}$ have some entries $\mathcal{M}_{i}(x, y)$ replaced by 0 when $x$ and $y$ are no longer connected by a flip, and possibly larger diagonal coefficients in order to insure that sums on columns are still equal to 1 . The following result will be of great use in the following, since it interconnects the four fibrations:

$$
\mathcal{M}=\frac{\mathcal{M}_{1}+\mathcal{M}_{2}+\mathcal{M}_{3}+\mathcal{M}_{4}}{3}-\frac{\mathrm{Id}}{3}
$$

where Id is the identity matrix. Indeed, each coefficient $\mathcal{M}(x, y)$ appears in all four matrices $\mathcal{M}_{i}$ but one, since the corresponding flip involves 3 de Bruijn lines. The last term of the sum insures that $\mathcal{M}$ is indeed a transition matrix. 
Note that $\mathcal{M}$ as well as $\mathcal{M}_{i}$ are symmetric matrices. Therefore their spectrum is real and they have an orthonormal eigenbasis. These two points will turn out to be quite useful in the following.

\section{Mixing times of octagonal tilings}

Our strategy in this central section is as follows: (i) we implement the above coupling technique in the case of octagonal tilings. More precisely, we construct a coupling technique on each fiber, then breaking the symmetry between the four de Bruijn families, since one family is arbitrarily chosen among 4 in the fibration viewpoint; (ii) couplings are then studied numerically; (iii) at the end of the section, we restore the lost symmetry using equation (8) to relate the dynamics on the whole lattice $L$ with dynamics on individual fibers. We also discuss the possible existence and the effect of "slower" fibers.

\subsection{Mixing times on a fiber}

The average coupling time on a fiber is estimated numerically. Note that, anticipating an extension of previous results on hexagonal tilings $[17,16]$, we expect the coupling time to behave like a power law of the system size, up to some possible logarithmic corrections. Note also that numerical techniques usually have some difficulties in exhibiting such logarithmic corrections: this point will deserve a careful analysis.

\subsubsection{Couplings on a fiber}

Our point of view is quite similar to the "routing" method developed in references $[15,16]$. The bijection between octagonal tilings and families of paths on hexagonal tilings has been previously introduced in section 2. On a fiber, the base tiling is fixed whereas the de Bruijn lines of the remaining family can flip (see figure 3).

To begin with, let us suppose that there is only one line in the latter family, denoted by $\ell$. As in references $[15,16]$, we first slightly modify the Markov chain in order to define a suitable coupling: at each step, we choose uniformly an internal point of $\ell$, the $i$-th vertex (starting from the left), as well as a number $r \in[0,1]$. If the $i$-th vertex is flippable upward (resp. downward) and $r \leq 1 / 2$ (resp. $r>1 / 2$ ), then we flip it. Note that, as compared to the original dynamics $\mathcal{M}$ (or $\mathcal{M}_{i}$ ), this dynamics has a different time scale: on the one hand, it is faster since the vertex to be flipped is chosen a priori on $\ell$. On the other hand, it is slowed down by a factor 2 since a feasible flip is actually realized with probability $1 / 2$, depending on $r$. However this modification does not change the nature of the fixed point or of the dynamics since it only implies a rescaling of the time unit.

As far as couplings are concerned, the order relation between lines is the following: a line $\ell_{1}$ is greater than another one $\ell_{2}\left(\ell_{1} \succeq \ell_{2}\right)$ if $\ell_{1}$ is entirely (but not strictly) above $\ell_{2}$. Figure 4 (Left) provides an example. Note that, as compared to the "routing" point of view of [16], we allow lines to cover (overlap) locally, since we have contracted de Bruijn lines of the corresponding family. The maximum (resp. minimum) configuration clearly lies on the top (resp. bottom) boundary of the hexagonal domain. If the two flips on $\ell_{1}$ and $\ell_{2}$ occur with same $i$ and $r$ and if $\ell_{1} \succeq \ell_{2}$ then their images satisfy the same relation. Indeed, as in reference [15], by construction - namely the introduction of $r$ - if a flip could bring $\ell_{1}$ below $\ell_{2}$, then the same flip would also apply to $\ell_{2}$, thus preserving the order between lines. As a consequence the coupling is monotonous.

In the general case with $p$ non-intersecting lines in each configuration ( $p=2$ in figure 4 , right), let us denote by $\ell_{i}^{(j)}, j=1, \ldots, p$, the $p$ lines of each configuration $\gamma_{i}$. Then $\gamma_{1} \succeq \gamma_{2}$ if for any $j, \ell_{1}^{(j)} \succeq \ell_{2}^{(j)}$. The configuration $\gamma$ is maximum (resp. minimum) when each of its lines is maximum (resp. minimum). As 

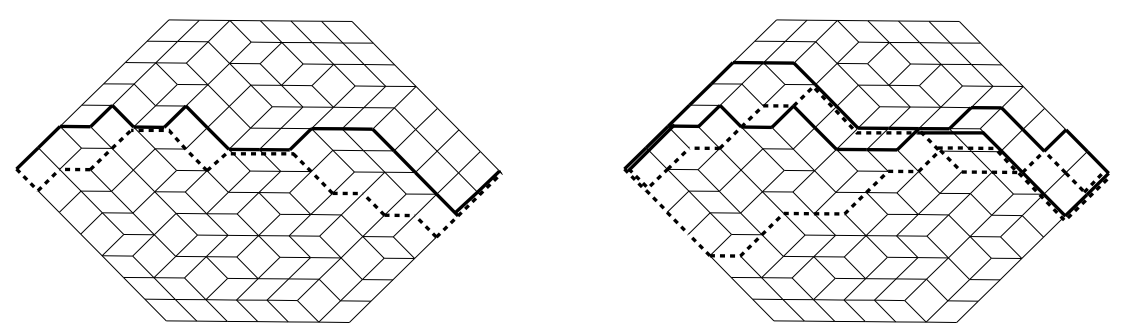

Fig. 4: Left: single-line coupling on a fiber. Right: multi-line coupling.

compared to the $p=1$ case, at each step, the index $j$ of the line to be flipped is chosen randomly between 1 and $p$, the same $j$ for $\gamma_{1}$ and $\gamma_{2}$. This also leads to a valid monotonous coupling.

\subsubsection{Heuristic argument}

With the underlying idea that couplings in paths-on-tilings problems do not differ qualitatively from couplings in a problem (of similar size) of paths on a square grid, we expect the following average coupling times: for a single-line coupling on a base tiling of sides of order $k, T \sim k^{3} \ln k$; for a $p$-line coupling, $T \sim k^{4} \ln k$. Indeed, D.B. Wilson predicts a similar result for hexagonal tilings [17] and therefore in the case of paths on a square grid (even in the case of single flips dynamics). Note however that we can already predict that the numerical prefactors in this asymptotic law will differ between hexagonal and octagonal cases; even if we ignore details of the local dynamics, for a given $n$, the distance (in number of flips) between extremal configurations is bigger in the octagonal case than in the hexagonal one, leading to a bigger prefactor in the former case. The two followings paragraphs are devoted to the quantitative numerical study of this point.

\subsubsection{Mixing times of single-line couplings}

To begin with, we study the case $p=1$, when there is only one de Bruijn line in each component of the coupling. We also suppose in a first time that the hexagonal base tilings are diagonal: all their sides are equal to $k$. Following reference [17], we expect $T$ to be asymptotic to $k^{3} \ln k$, up to a numerical prefactor.

For a given base hexagonal tiling $\mathcal{T}_{a}$ associated with the fiber $J_{a}$, we run a number $m$ of couplings until they coalesce, and then estimate the coupling time $T\left(\mathcal{T}_{a}\right)$. We then make a second average on $M$ different tilings, in order to get the time $\bar{T}$ averaged on tilings $\mathcal{T}_{a}$. We also keep track of the standard deviation of $T, \Delta T$. From numerical data (see figure 5), we draw the following conclusions:

- $\Delta T / \bar{T}$ seems to decrease toward a constant $(\simeq 0.07)$ as $k \rightarrow \infty$, which means that the average coupling time $T\left(\mathcal{T}_{a}\right)$ goes on depending on the base tiling $\mathcal{T}_{a}$ at the large size limit. However, most $T\left(\mathcal{T}_{a}\right)$ are of order $\bar{T}$, and the mixing times $\tau(\varepsilon)$ on most fibers are controlled by $\bar{T}$. Nevertheless, the effect of few "slower" fibers will deserve a detailed discussion below (section 3.3.2).

- The measures of $T\left(\mathcal{T}_{a}\right)$ are compatible with a $k^{3} \ln k$ behavior, as figure 5 illustrates: the graph $\bar{T}$ versus $k^{3} \ln k$ is linear up to error bars. In particular, this fit with logarithmic corrections is much better than a simple power-law fit. The slope is equal to $25.51 \pm 0.05$. 


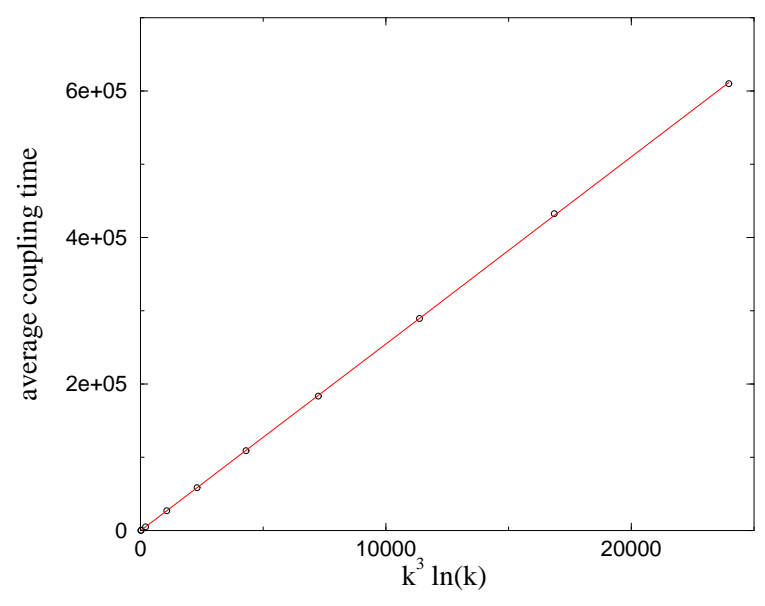

Fig. 5: In the case of 1-line systems, numerical estimates of $\bar{T}$ as a function of $k^{3} \ln k$ (circles) up to $k=20$, and linear fit. Error bars are smaller than the size of symbols.

\subsubsection{Mixing times of multi-line couplings; Non-diagonal base tilings}

We still work in the diagonal case where the 3 sides of the base hexagonal tilings are equal to $k$. Moreover, $p=k$. We expect $T \sim C s t . k^{4} \ln k$ [17]. The same conclusions as in the previous one-line case hold (see figure 6). The limit of $\Delta T / \bar{T}$ is also about 0.07. Moreover, the slope is equal to $42.79 \pm 0.11$.

We also have explored non-diagonal cases where all the sides of the hexagonal boundary are not necessarily equal. For example, single-line couplings on hexagonal base tilings of sides $(k, 2 k, 3 k)$ also display a $k^{3} \ln k$ asymptotic behavior with a slope $346.1 \pm 1.7$. However in this last case, $\Delta T / \bar{T}$ is larger than in the previous diagonal ones, since it is close to 0.2 .

As a conclusion, we get a rather natural result: couplings in fibers seem to behave like couplings in hexagonal tiling problems, up to different numerical prefactors: the dynamics on each fiber is rapidly mixing. In the next section, we return to the dynamics on the whole set of tilings and we demonstrate how to restore the symmetry that was lost in the fibration process.

\subsection{Large-time rates of convergence and second eigenvalues}

In figure 7 , we have schematically represented the configuration space of the tilings filling an octagon of sides 1,1,1 and 2. We have also represented two fibrations (among four) on this lattice. Examining this figure, one remarks that the two fibrations are to a certain extent "transverse": one can connect any two configurations using flips of only two fibers; in appendix A, we generalize this result to any tiling set. If the dynamics is rapidly mixing in each fiber, the combination of dynamics on two (and even four) fibrations will certainly also be rapidly mixing. Beyond this heuristic argument, it is possible to related rigorously dynamics in $L$ and dynamics in fibers, at least their large time behavior. This section, together with appendix $\mathrm{B}$, is dedicated to this point.

It is common in the field of Markov processes to relate rates of convergence to spectra of transition matrices. Generally speaking, let $M$ be a transition matrix. Then 1 is always the largest eigenvalue in modulus, and the difference between 1 and the second largest eigenvalue will be called the first gap of 


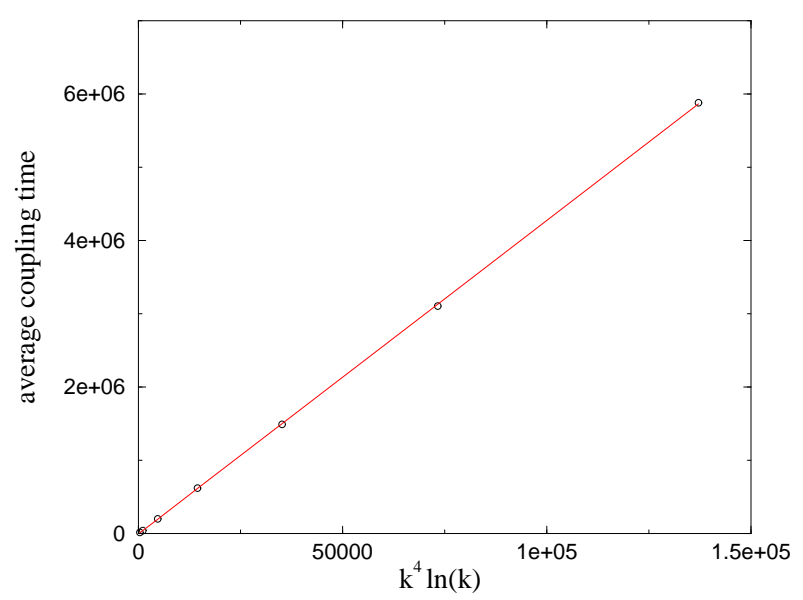

Fig. 6: In the case of $p$-line systems, numerical estimates of $\bar{T}$ as a function of $k^{4} \ln k$ (circles), up to $k=15$, and linear fit. Error bars are smaller than the size of symbols.

$M$, and denoted by $g(M)$. Then the mixing time $\tau(\varepsilon)$ is monitored by this gap: roughly speaking, for sufficiently small $\varepsilon$, or equivalently for sufficiently large times,

$$
\frac{\ln (1 / \varepsilon)}{2 g(\mathcal{M})} \leq \tau(\varepsilon) \leq \frac{\ln (1 / \varepsilon)}{g(\mathcal{M})}
$$

We shall discuss the validity and the limits of such a relation in the following section 3.3.1.

Moreover, we prove in appendix B the following central "gap relation":

$$
g(\mathcal{M}) \geq \inf _{i}\left(g\left(\mathcal{M}_{i}\right)\right)
$$

which, owing to relation (9), precisely means that the large-time dynamics in $L$ is at least as fast as the dynamics in the slowest fibration: if $\tau_{i}(\varepsilon)$ denotes mixing times associated with matrices $\mathcal{M}_{i}$, then

$$
\tau(\varepsilon) \leq 2 \sup _{i} \tau_{i}(\varepsilon)
$$

Since the dynamics in fibration $i$ is dominated by its slowest fiber, one finally gets that the dynamics in $L$ is at least as fast as the dynamics in the slowest fiber (up to a factor 2). This result finally restores the symmetry lost when choosing a de Bruijn family among the four possible ones.

\subsection{Discussions}

\subsubsection{Short-time convergence rates}

In the previous section, we have used equation (9) to relate the gaps of transition matrices and mixing times. However, strictly speaking, the exact relation is

$$
\frac{\mu_{1} \ln (1 / 2 \varepsilon)}{2 g(\mathcal{M})} \leq \tau(\varepsilon) \leq \frac{\ln (1 / \varepsilon)+\ln (N)}{g(\mathcal{M})},
$$




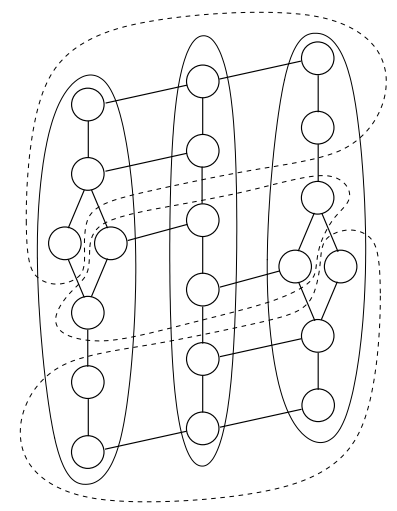

Fig. 7: The lattice of tilings filling an octagon of sides 1,1,1 and 2. The complete lattice can be found in reference [6]. Two fibrations among four are represented. They are equivalent because in both cases de Bruijn lines of the chosen family play a symmetric role.

where $\mu_{1}$ is the second largest eigenvalue and $N$ denotes the number of configurations $[29,15]$. Since $N$ grows exponentially with the number of tiles $N_{T}$, one gets

$$
\tau(\varepsilon) \leq \frac{\ln (1 / \varepsilon)+S N_{T}}{g(\mathcal{M})}
$$

where $S$ is an entropy per tile [7]. Moreover $\mu_{1}$ tends to 1 at the large size limit. Therefore, relation (9) becomes asymptotically exact when $\varepsilon$ tends to 0 since $\ln (1 / \varepsilon)$ becomes large as compared to $N_{T}$.

But, as discussed for instance in reference [17], the main motivation of computing mixing times is not only to know large time behaviors (or equivalently small $\varepsilon$ behaviors) of Markovian processes but also to know how many flips are necessary to get a configuration close to random. In practice, this means that the variation distance (1) need be small, but not unnecessarily small, say some percents. Therefore we also would like to know the short-time behavior of the variation distance (1). For many Markovian processes [17], when the distance to equilibrium is measured by the above variation distance (1), there is a threshold phenomenon: the distance stays close to 1 for a more or less long period, then drops rapidly and enters an asymptotic exponential regime. In this case

$$
\tau(\varepsilon) \approx \tau_{t h .}+\frac{\ln (1 / \varepsilon)}{g(\mathcal{M})},
$$

where $\tau_{t h}$. is the above threshold. The upper bound in (12) matches this form, whereas the upper bound provided by the coupling technique does not, and might certainly be refined. In particular, the coupling time $T$ provides an upper bound for $\tau_{t h}$. [17].

As a consequence, the knowledge of the gap $g(\mathcal{M})$ is not sufficient to derive a tight upper bound for $\tau(\varepsilon)$ at short times. However, as detailed below, relation (13) is sufficient to derive a polynomial upper bound for $\tau(\varepsilon)$ and to prove that flip dynamics are rapidly mixing. 


\subsubsection{Statistics and effect of rare "slow" fibers}

In the previous sections, we have proved that the coalescence times $\bar{T}$ averaged on both realizations of Markovian coupled processes and different fibers of a same fibration behave like $k^{4} \ln k$ in diagonal cases. Since the number of tiles is $N_{T}=6 k^{2}$, we finally get

$$
\bar{T} \simeq \kappa_{4} N_{T}^{2} \ln \left(N_{T}\right)
$$

where $\kappa_{4}=1.189 \pm 0.003$ in the original Markov chain time unit (see discussion about time units in paragraph 3.1.1). However, the coupling time $T$ depends on the associate fiber and the distribution of times $T$ in a given fibration have a certain width, as it is illustrated in figure 8. Now our proof is based

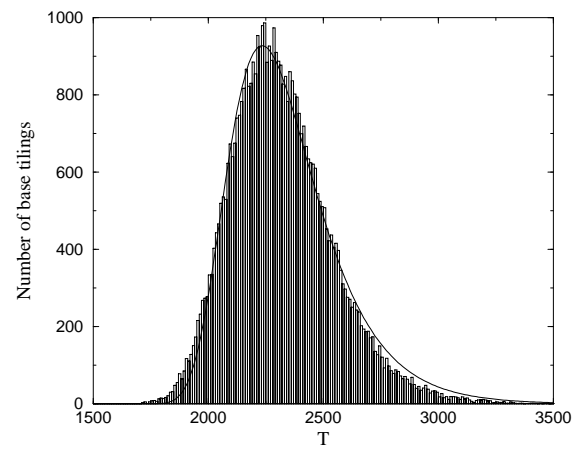

(a)

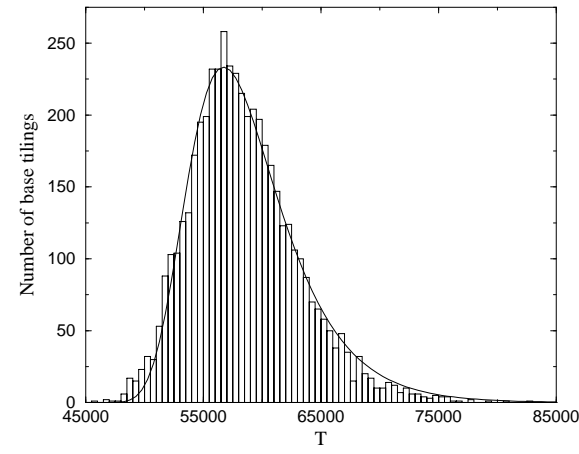

(b)

Fig. 8: Numerical distributions of coupling times $T$ around the average value $\bar{T}$ for a diagonal base tiling and for different values of $k$ : (a): $k=4$; (b): $k=10$. In order to get more samples, we focus on single-line couplings: the sizes of the samples are respectively equal to 47800 (among about 230000 tilings) and 4880 . The continuous curves are Gumbel distributions fitting the numerical data.

upon tracking the highest eigenvalues. The slowest fibers will dominate the dynamics on the fibration $i$, the gap of $\mathcal{M}_{i}$ and therefore the whole dynamics on $L$. However the above distributions, even though wide, are monodisperse, as illustrated in the figure, and have a width $\Delta T$ comparable to their average value $\bar{T}$ as discussed above. Therefore the typical values of coupling times should remain of the order of magnitude of the average value (15).

Nevertheless, the above arguments do not exclude definitively the existence of rare slow fibers in the upper tails of these distributions. We propose two arguments to treat this question of slow fibers. The first one is the less rigorous but provides a better upper bound. It is developed in appendix C.

The second argument is based upon a careful analysis of numerical distributions of times $T\left(\mathcal{T}_{a}\right)$. We recall that coupling times $T$ are maxima of coalescence time distributions (see relation (5)). Therefore the expected shape of these numerical distributions ought to be sought in the specific class of distributions of extrema of families of random variables, namely Gumbel distributions [30, 31]: we first recall some standard results of extreme-value statistics.

Consider $N$ independent and identically distributed random variables $T_{s}, s=1, \ldots, N$. We suppose that 
the probability densities decay rapidly at large $T$ :

$$
p(T) \simeq \frac{C_{1}}{T^{\alpha}} \exp \left(-C_{2} T^{\beta}\right),
$$

where all constants save $\alpha$ are positive. We define the variable under interest $T_{\max }=\sup _{s} T_{s}$. We have the following interesting result: at large $N$, the probability density of $T_{\max }$ satisfies

$$
p(u)=\exp (-u-\exp (-u))
$$

where $u=\left(T_{\max }-T_{0}\right) / \delta T$ is a suitably rescaled variable: $T_{0}$ is the most probable value of $T_{\max }$ and $\delta T$ measures the width of the distributions. This universal form is independent of the constants $C_{1}, C_{2}, \alpha$ or $\beta$.

As far as our numerical data are concerned, the random variables $T_{s}$ are all the coalescence times on a given base tiling $\mathcal{T}_{a}$, associated with an initial configuration $\left(x_{0}, y_{0}\right)$ on the tiling $\mathcal{T}_{a}\left(s=\left(\mathcal{T}_{a}, x_{0}, y_{0}\right)\right)$, and averaged over realizations of the coupled Markov process: $T_{s}=\left\langle T\left(x_{0}, y_{0}\right)\right\rangle$. The extreme-value variables are the coupling times $T=\sup _{\left(x_{0}, y_{0}\right)} T_{s}$.

Therefore the variables $T_{s}$ are not strictly speaking identically distributed. They are not independent either since they are associated with the same base tiling $\mathcal{T}_{a}$. Nevertheless, up to slight discrepancies, our numerical distributions appear to be very well fitted by this kind of distribution, as illustrated in figure 8 . This result is not surprising since, as stated above, the times $T$ are maxima of random variables. It was numerically tested that $T_{0}$ and as well as $\delta T \propto \Delta T$ grow like $k^{4} \ln k$ in diagonal cases.

The interest of this result is that it provides the large $T$ behavior of coupling time distributions. As a consequence we are able to estimate the largest coupling time, which will prove to be sufficient to give a polynomial upper bound to mixing times on fibers and therefore to prove that dynamics on tiling sets are rapidly mixing. Let us focus on diagonal cases: $T_{0}$ as well as $\delta T$ behave like $k^{4} \ln k$. At large $T, p\left(T_{\max }\right)$ is dominated by an exponential decay: $p\left(T_{\max }\right) \sim \exp (-u) \sim \exp \left(-T_{\max } / \delta T\right)$. Moreover, for a side-length $k$ and a fibration $i$, there are $N_{i}$ base tilings $\mathcal{T}_{a}$. Therefore if $T^{*}$ denotes the larger coupling time on all these different tilings, it is estimated by

$$
N_{i} \exp \left(-T^{*} / \delta T\right) \approx 1
$$

Now $N_{i}$ is a number of tilings: it grows exponentially with the area of the tiling, that is with $k^{2}: \ln N_{i} \approx$ Cst. $k^{2}$. Thus $T^{*} \approx C s t . k^{2} \delta T$, and

$$
T^{*} \approx C s t .^{\prime} k^{6} \ln k \approx C s t .{ }^{\prime \prime} N_{T}^{3} \ln N_{T} .
$$

This largest coupling time provides a more rigorous upper bound than the pruning process of appendix C. Namely, putting together this upper bound for the slowest fiber and relations (12) and (13), one gets, for all $\varepsilon$ :

$$
\tau(\varepsilon) \leq 2 S C s t . .^{\prime \prime} N_{T}^{4} \ln N_{T}+2 C s t .^{\prime \prime} N_{T}^{3} \ln N_{T} \ln (1 / \varepsilon) .
$$

Flip dynamics in octagonal tiling sets are rapidly mixing.

However the arguments of appendix $\mathrm{C}$ suggest that rare slow fibers should not be relevant, and that one should rather focus on typical coupling times on fibers, leading to the following upper bound for mixing times of octagonal tilings at large times:

$$
\tau(\varepsilon) \leq 2 C \text { e } N_{T}^{2} \ln N_{T} \ln (1 / \varepsilon),
$$


where $C$ is a constant of order $\kappa_{4}$. We should also mention that coupling techniques do not necessary provide the best upper bounds [26] and that mixing times might even be shorter than (21) (see in particular the discussion of section 3.3.1).

Moreover, the argument at the beginning of section 3.2 suggests that mixing times in the whole set $L$ are of order of magnitude of mixing times in fibrations. Now the $N_{T}^{2} \ln N_{T} \ln (1 / \varepsilon)$ mixing times in fibers are valid even at short times. As a consequence, we believe that the above upper bound (21) should also be valid at short times.

\section{Higher symmetry plane tilings}

In this section, we discuss what the above result becomes in the case of two-dimensional tilings of larger symmetries filling $2 D$-gons. These tilings are made of rhombi with angles multiple of $\pi / D$ (see $[7,14]$ ) and they are also endowed with 3-tile elementary flips which still connect the set of configurations.

All the above approach can be extended to higher-symmetry tilings. Some points need to be clarified further. The fibration concept is general [19]: there are $D$ fibrations associated with $D$ de Bruijn families. Moreover, as far as coupling times on fibers are concerned, our phenomenological arguments remains valid in this case. We also have tested coupling times on fibers numerically. For example, for $D=5$ coupling times on fibers still behave as in the octagonal case: in the diagonal cases of side $k$ with $k$ line couplings, we still find that coupling times are asymptotic to $N_{T}^{2} \ln N_{T}$, up to a different numerical prefactors $\kappa_{5}=2.80 \pm 0.10$ in the original time unit. We also note that Gumbel distributions remain the good description of coupling time distributions but that $\Delta T / \bar{T}$ seems to be larger in this case, since it is of order 0.2 when the base tilings are diagonal.

The only point to be checked in detail is that a gap relation like (10) still holds for larger $D$. Relation (8) becomes

$$
\mathcal{M}=\frac{\mathcal{M}_{1}+\mathcal{M}_{2}+\ldots+\mathcal{M}_{D}}{3}-\frac{D-3}{3} \mathrm{Id},
$$

with evident notations. We also define $\tilde{\mathcal{M}}=\left(\mathcal{M}_{1}+\ldots+\mathcal{M}_{D}\right) / D$ : section 3.2 and appendix B can easily be adapted and one gets

$$
g(\tilde{\mathcal{M}}) \geq \frac{D-1}{D} \inf _{i=1, \ldots, D}\left(g\left(\mathcal{M}_{i}\right)\right)
$$

and

$$
g(\mathcal{M}) \geq \frac{D-1}{3} \inf _{i=1, \ldots, D}\left(g\left(\mathcal{M}_{i}\right)\right) .
$$

The dynamics on the whole set is faster by a factor $(D-1) / 3$ than the dynamics on the slowest fibration. Therefore in relation (21), the constant $C$ is of order the constant $\kappa_{D}$ rescaled by a factor $3 /(D-1)$ (see table 1).

However, when $D$ increases, numerical measures are less and less precise. Indeed, the widths of the Gumbel distributions increase with $D$ and the measures of these widths become imprecise themselves. Technically speaking, because of their exponential decay at large $T$, the distributions have a large forth moment, which prevents a precise measure of $\Delta T$. Therefore it seems difficult to estimate correctly $\kappa_{D}$ as well as the error on $\kappa_{D}$ in reasonable computational time beyond $D=6$.

At last, note that this approach is not valid for $D=3$ since in that case there is a unique fiber in each fibration that coincides with the whole configuration set and relation (34) does not hold. 
Tab. 1: Numerical constants $3 \kappa_{D} /(D-1)$. For diagonal tilings of symmetry $2 D$, the constant $C$ in relation (21) is of order $3 \kappa_{D} /(D-1)$.

\begin{tabular}{|l|r|r|r|}
\hline$D$ & 4 & 5 & 6 \\
\hline \hline$\frac{3 \kappa_{D}}{(D-1)}$ & $1.189 \pm 0.003$ & $2.10 \pm 0.08$ & $\approx 4$ \\
\hline
\end{tabular}

\section{General conclusion}

We have established that random tilings of rhombi with fixed octagonal boundary conditions endowed with local 3-tile rearrangements of tiles are rapidly mixing. Our analysis is based upon a subtle decomposition scheme of the configuration set in smaller subsets on which it is possible to develop a coupling technique. This decomposition is followed by a "reconnection" process which restores the initial symmetry lost in the decomposition process. We conclude that mixing times are bounded above by a power-law of the system size, up to logarithmic corrections. Note however that this approach certainly misses tight upper bounds for at least two reasons: on the one hand, the coupling technique as well as the relation bewteen transition matrix gaps and mixing times provide correct upper bounds but not tight ones at short times. On the other hand, the pruning process in the case where several pruned fibers intersect should also be refined, in order to justify tighter upper bounds at all times. These two points will need further clarification.

We also generalized this approach to larger symmetry tilings (with $2 D$-gonal boundaries) and our conclusions remain identical. However numerical prefactors for larger $D$ systems should be considered with attention, in particular their large $D$ asymptotic behavior. Indeed these prefactors seem to increase with $D$. Understanding why they increase whereas the local structure of larger $D$ tilings does not essentially differ from its hexagonal or octagonal counterpart is a challenging task. In particular, the role of large coordination vertices - which only appear in large $D$ tilings - should be analyzed in detail: it is very long to destroy such local structures since their coordination number must be reduced to 3 by flips on neighboring vertices before they can be flipped in their turn. The reason why $\Delta T / \bar{T}$ also increases with $D$ should be related to this point as well, since dynamics on rare base tilings with a "large" amount of large coordination vertices must be particularly slow.

The question of slow dynamics in rhombus random tilings in the presence of energetic interactions between tiles should also be addressed in the frame of this analysis: do they remain rapidly mixing, or can sufficiently many configurations appear which significantly slow down the dynamics [32, 33]? Using the same construction as in the paper, if the dynamics happen to be rapid in fibers at finite temperature, the system will be rapidly mixing. Conversely, the existence of slow dynamics should be detectable in fiber dynamics, even though, as it was discussed in this paper, the existence of slow dynamics in some fibers does not involve their existence in the whole system. Moreover, note that relation (37) has been established in the case where all transition matrices are symmetric, which is false in the presence of energetic interactions. Therefore the methods of the present paper cannot be generalized without care at finite temperature.

At last, what does the present analysis become in the case of larger dimension tilings, related for example to quasicrystals with icosahedral symmetry? Even if the fibration process remains valid, it should be considered with care since the connectivity of fibers has not even been establish with certainty [19]. 
Moreover, configurations in fibers cannot be represented by routings any longer and all the machinery which leads to a valid coupling technique remains to be built. This point is also a challenging open question.

\section{Acknowledgements}

I am indebted to M. Latapy, K. Frahm, R. Mosseri, D.S. Dean for useful discussions and comments on this work. I am also grateful to L. Orozco who checked the gap relation for small systems as part of his Master's project.

\section{A Fibers always intersect in octagonal tiling sets}

In an octagonal tiling set, given two fibers $J_{i, a}$ and $J_{j, b}$ of fibrations $i$ and $j$, we prove that they always have a non empty intersection. Or equivalently, that given two tilings $A$ and $B$ which belong respectively to the fiber $J_{i, a}$ of fibration $i$ and to the fiber $J_{j, b}$ of fibration $j$, there exists a sequence of flips in fibers $J_{i, a}$ and $J_{j, b}$ exclusively which connect $A$ to $B$.

In section 2.3, we have defined de Bruijn families. For an octagonal tiling, there are 4 such families. Staying in a fiber $J_{i, a}$ means performing only flips involving a line of the de Bruijn family $i$. Since our tilings fill an octagon, any two lines of two different families always intersect. Now, topologically speaking, a tiling is defined by the relative intersections of the 4 families. It is the so-called de Bruijn dual representation of tilings. We shall not describe this representation in detail since the reader can already find it in many papers (for example [34, 20]). In this representation - or even in the original tiling representation! - it is easy to understand that one can go from $A$ to $B$ by flipping in a first time only lines of family $i$ and moving them to their right position, then flipping only lines of family $j$.

Note that following the same line, one can prove that in a $2 D$-fold symmetry tiling, one can connect any two tilings with flips belonging to $D-2$ fibers only.

\section{B Proof of the gap relation}

We derive in this appendix a proof of the gap relation (10). Instead of $\mathcal{M}$, we will work with the matrix

$$
\tilde{\mathcal{M}}=\frac{\mathcal{M}_{1}+\mathcal{M}_{2}+\mathcal{M}_{3}+\mathcal{M}_{4}}{4}=\frac{3}{4} \mathcal{M}+\frac{1}{4} \mathrm{Id},
$$

which presents the advantage of having non-negative eigenvalues. Indeed each diagonal coefficient of $\mathcal{M}_{i}$ is larger than $1 / 2$. As a consequence, following a remark of reference [15], $\mathcal{M}_{i}$ has a non-negative spectrum. The same remark holds for $\tilde{\mathcal{M}}$. At the end of the calculation, the gap of $\mathcal{M}$ will simply be: $g(\mathcal{M})=4 / 3 g(\tilde{\mathcal{M}})$.

Given a state $e$, we denote by $e^{\text {eq }}\left(\right.$ resp. $\left.e_{i}^{\text {eq }}\right)$ its projections on the eigenspaces of $\tilde{\mathcal{M}}$ (resp. $\mathcal{M}_{i}$ ) associated with the eigenvalue $\lambda=1$ (the superscript "eq" stands for "equilibrium"). If $N$ still denotes the number of configurations, the vector $e^{\text {eq }}=(1 / N, \ldots, 1 / N)$ is independent of $e$, whereas $e_{i}^{\text {eq }}$ depends on $e$ and $i$, since the eigenspace associated with $\lambda=1$ is degenerate. All the difficulty in the following dwells in this degenerate character.

We suppose now that we have sorted altogether all the eigenvalues of the 4 matrices $\mathcal{M}_{i}: 1=\lambda>\mu_{1} \geq$ $\mu_{2} \geq \ldots \geq \mu_{q} \geq 0$. We denote by $f_{j}$ the normalized eigenstate associated with the eigenvalue $\mu_{j} ; f_{j}$ can $a$ 
priori be the eigenstate of any matrix $\mathcal{M}_{i}$. We write

$$
\begin{aligned}
e-e^{\mathrm{eq}} & =\frac{1}{4} \sum_{i=1}^{4}\left(e-e_{i}^{\mathrm{eq}}\right)+\frac{1}{4} \sum_{i=1}^{4}\left(e_{i}^{\mathrm{eq}}-e^{\mathrm{eq}}\right) \\
& =\frac{1}{4} \sum_{j=1}^{q} \alpha_{j} f_{j}+\frac{1}{4} \sum_{i=1}^{4}\left(e_{i}^{\mathrm{eq}}-e^{\mathrm{eq}}\right)
\end{aligned}
$$

where each vector $\left(e-e_{i}^{\mathrm{eq}}\right)$ has been projected on the eigenbasis of $\mathcal{M}_{i}$, and

$$
\begin{aligned}
\tilde{\mathcal{M}}\left(e-e^{\mathrm{eq}}\right) & =\frac{1}{4} \sum_{i=1}^{4} \mathcal{M}_{i}\left(e-e^{\mathrm{eq}}\right) \\
& =\frac{1}{4} \sum_{i=1}^{4} \mathcal{M}_{i}\left(e-e_{i}^{\mathrm{eq}}\right)+\frac{1}{4} \sum_{i=1}^{4}\left(e_{i}^{\mathrm{eq}}-e^{\mathrm{eq}}\right) \\
& =\frac{1}{4} \sum_{j=1}^{q} \mu_{j} \alpha_{j} f_{j}+\frac{1}{4} \sum_{i=1}^{4}\left(e_{i}^{\mathrm{eq}}-e^{\mathrm{eq}}\right),
\end{aligned}
$$

by definition of $f_{j}$ and $\mu_{j}$. Now, thanks to a suitable Abel transform,

$$
\begin{aligned}
\tilde{\mathcal{M}}\left(e-e^{\mathrm{eq}}\right) & =\frac{1}{4}\left(1-\mu_{1}\right) \sum_{i=1}^{4}\left(e_{i}^{\mathrm{eq}}-e^{\mathrm{eq}}\right) \\
& +\frac{1}{4} \sum_{k=1}^{q-1}\left(\mu_{k}-\mu_{k+1}\right)\left(\sum_{i=1}^{4}\left(e_{i}^{\mathrm{eq}}-e^{\mathrm{eq}}\right)+\sum_{j=1}^{k} \alpha_{j} f_{j}\right) \\
& +\frac{1}{4} \mu_{q}\left(\sum_{i=1}^{4}\left(e_{i}^{\mathrm{eq}}-e^{\mathrm{eq}}\right)+\sum_{j=1}^{q} \alpha_{j} f_{j}\right) .
\end{aligned}
$$

Thus, if $\|\cdot\|$ stands for the Euclidean norm,

$$
\begin{aligned}
\left\|\tilde{\mathcal{M}}\left(e-e^{\mathrm{eq}}\right)\right\| & \leq\left(1-\mu_{1}\right)\left\|\frac{1}{4} \sum_{i=1}^{4}\left(e_{i}^{\mathrm{eq}}-e^{\mathrm{eq}}\right)\right\| \\
& +\sum_{k=1}^{q-1}\left(\mu_{k}-\mu_{k+1}\right)\left\|e-e^{\mathrm{eq}}\right\|+\mu_{q}\left\|e-e^{\mathrm{eq}}\right\| . \\
& \leq\left(1-\mu_{1}\right)\left\|\frac{1}{4} \sum_{i=1}^{4}\left(e_{i}^{\mathrm{eq}}-e^{\mathrm{eq}}\right)\right\|+\mu_{1}\left\|e-e^{\mathrm{eq}}\right\| .
\end{aligned}
$$

Indeed, $\mu_{k}-\mu_{k+1} \geq 0$ and $\mu_{q} \geq 0$; moreover, for any $k, \sum_{i=1}^{4}\left(e_{i}^{\mathrm{eq}}-e^{\mathrm{eq}}\right)+\sum_{j=1}^{k} \alpha_{j} f_{j}$ is the sum of 4 orthogonal projections of $e-e^{\mathrm{eq}}$ on suitable spaces, the norm of each of them being therefore smaller than $\left\|e-e^{\mathrm{eq}}\right\|$. Moreover, we shall prove below that for large tilings

$$
\left\|\sum_{i=1}^{4}\left(e_{i}^{\mathrm{eq}}-e^{\mathrm{eq}}\right)\right\| \leq\left\|e-e^{\mathrm{eq}}\right\|
$$


As a consequence,

$$
\left\|\tilde{\mathcal{M}}\left(e-e^{\mathrm{eq}}\right)\right\| \leq\left[\frac{1}{4}\left(1-\mu_{1}\right)+\mu_{1}\right]\left\|e-e^{\mathrm{eq}}\right\|
$$

from which it follows that

$$
g(\tilde{\mathcal{M}}) \geq \frac{3}{4}\left(1-\mu_{1}\right) \geq \frac{3}{4} \inf _{i}\left(g\left(\mathcal{M}_{i}\right)\right) .
$$

Thus

$$
g(\mathcal{M}) \geq \inf _{i}\left(g\left(\mathcal{M}_{i}\right)\right)
$$

Now we establish relation (34). More precisely, we demonstrate it in the large system size limit, which rigorously establishes gap relation (37) in this limit of interest. However, we also checked "by hand" this relation in several small systems, namely in the cases of octagons of sizes: $(1,1,1,1),(1,1,1,2),(1,1,1,3)$, $(1,1,1,4),(1,1,1,7),(1,2,1,2),(1,2,1,3),(1,3,1,3),(1,2,1,5),(1,1,2,2)$, and $(1,2,2,2)$. A complete proof should be writable, but it would require unessential complicated calculations. Our proof relies on very simple Euclidean geometry arguments.

We shall prove that at the large size limit, the four vectors $\left(e_{i}^{\mathrm{eq}}-e^{\mathrm{eq}}\right)$ form an orthogonal system. Since they are orthogonal projections of $\left(e-e^{\mathrm{eq}}\right)$ onto orthogonal directions, it will follow that their sum has a smaller norm than $\left(e-e^{\mathrm{eq}}\right)$. Therefore we will demonstrate that the cosine of the angle between two such vectors of indices $i$ and $j$,

$$
\cos \left(\theta_{i j}\right)=\frac{\left(e_{i}^{\mathrm{eq}}-e^{\mathrm{eq}}\right)}{\left\|e_{i}^{\mathrm{eq}}-e^{\mathrm{eq}}\right\|} \cdot \frac{\left(e_{j}^{\mathrm{eq}}-e^{\mathrm{eq}}\right)}{\left\|e_{j}^{\mathrm{eq}}-e^{\mathrm{eq}}\right\|}
$$

tends to 0 at the large size limit.

Since $\left(e_{i}^{\mathrm{eq}}-e^{\mathrm{eq}}\right)$ is constant on each fiber $J_{i, a}$ of the fibration $i$, we can decompose it on the family of vectors $g_{i, a}$ constant on fibers: $g_{i, a}=0$ on each component except on the fiber $J_{i, a}$ where it is equal to $1 /\left|J_{i, a}\right|$, where $|J|$ stands for cardinality of set $J$. Then

$$
\frac{\left(e_{i}^{\mathrm{eq}}-e^{\mathrm{eq}}\right)}{\left\|e_{i}^{\mathrm{eq}}-e^{\mathrm{eq}}\right\|}=\sum_{a} \alpha_{i, a} \frac{g_{i, a}}{\left\|g_{i, a}\right\|},
$$

where $\sum_{a} \alpha_{i, a}^{2}=1$ (the $g_{i, a}$ 's are orthogonal for a given family $i$ ). The same condition holds for the vector $\left(e_{j}^{\mathrm{eq}}-e^{\mathrm{eq}}\right)$ and the fibration $j$.

Now, by definition of $g_{i, a}$ and $g_{j, b}$,

$$
\begin{aligned}
g_{i, a} \cdot g_{j, b} & =\frac{\left|J_{i, a} \cap J_{j, b}\right|}{\left|J_{i, a}\right|\left|J_{j, b}\right|} \\
\left\|g_{i, a}\right\|^{2} & =\frac{1}{\left|J_{i, a}\right|}
\end{aligned}
$$

Hence

$$
\begin{aligned}
\left|\cos \left(\theta_{i j}\right)\right| & \leq\left(\sum_{a, b}\left|\alpha_{i, a}\right|\left|\alpha_{j, b}\right|\right) \sup _{a, b} \frac{\left|J_{i, a} \cap J_{j, b}\right|}{\sqrt{\left|J_{i, a}\right|\left|J_{j, b}\right|}} \\
& \leq \sup _{a, b} \frac{\left|J_{i, a} \cap J_{j, b}\right|}{\sqrt{\left|J_{i, a}\right|\left|J_{j, b}\right|}}
\end{aligned}
$$


by Cauchy-Schwarz inequality. Without loss of generality, suppose that $\left|J_{i, a}\right| \leq\left|J_{i, b}\right|$. Then the last quantity is smaller than $\left|J_{i, a} \cap J_{j, b}\right| /\left|J_{i, a}\right|$. But this quantity is vanishingly small at the infinite size limit. Indeed, the fiber $J_{i, a}$ contains a given amount of intersections $J_{i, a} \cap J_{j, b}$ which grows like the number of transverse fibers $J_{i, b}$. Since this last number grows exponentially with the system size, the ratio $\mid J_{i, a} \cap$ $J_{j, b}|/| J_{i, a} \mid$ goes to 0 when the system size grows to infinity.

\section{Bypassing slow fibers: "pruning" process}

We shall argue in the following that rare slow fibers artificially increase upper bounds on mixing times and can actually be neglected when evaluating global dynamical rates. The underlying idea is that since there are 4 fibrations, slow fibers of a given fibration can be bypassed via faster fibers of other fibrations. We introduce a "fiber-pruning" process, which consists in getting rid of undesirable slow modes by setting to 0 all the transition probabilities between the elements of slow fibers $J_{i, a}$ in matrices $\mathcal{M}_{i}$. We argue below that one can prune as many fibers as needed provided the configuration set remains connected. The remaining modes being rapid, the above theory can be applied to prove that the whole Markovian process $\mathcal{M}$ is indeed rapidly mixing.

Given a fiber $J_{i, a}$, we say that we "prune" this fiber if in the matrix $\mathcal{M}_{i}$, all the transition probabilities between two different tilings of $J_{i, a}$ are set to 0 , while all the diagonal coefficients associated with tilings of $J_{i, a}$ are set to 1 . The 3 other matrices $\mathcal{M}_{j}, j \neq i$, remain unchanged. In other words, the flips inside the fiber $J_{i, a}$ are not allowed any longer.

Several fibers can be pruned in the same way, resulting in new matrices $\mathcal{M}_{i}^{\prime}$ and a new global matrix $\mathcal{M}^{\prime}$. Every off-diagonal coefficient of $\mathcal{M}^{\prime}$ is smaller than the corresponding element of $\mathcal{M}$, thus (see [35] for instance) the process associated with $\mathcal{M}^{\prime}$ is slower than the one associated with $\mathcal{M}$. If we prove that the former is rapidly mixing, then we will have established that the latter also is.

Let us first discuss rigorously what is the effect of pruning the slowest fiber, associated with the second largest eigenvalue $\mu_{1}$. We want to establish that the dynamics is actually monitored by the gap $1-\mu_{2}$.

All the configurations $x \in J_{i, a}$ have been isolated in the fibration $i$, which enables us to consider them as degenerate "fibers" $\{x\}$. The new matrix $\mathcal{M}_{i}$ does not have $\mu_{1}$ as eigenvalue any longer, and all the above argumentation of section 3.2 and appendix B can be adapted to this new fibration with the set of eigenvalues $1=\lambda>\mu_{2} \geq \ldots \geq \mu_{q} \geq 0$. The only difficulty is to adapt the proof of appendix B since the cardinalities of fibers have changed. The vectors $g_{i,\{x\}}$ are now constant and equal to 1 on $\{x\}$ and to 0 everywhere else. Hence if the fiber $J_{j, b}$ intersects the "fiber" $\{x\}$ then

$$
\begin{aligned}
g_{i,\{x\}} \cdot g_{j, b} & =\frac{1}{\left|J_{j, b}\right|}, \\
\left\|g_{i,\{x\}}\right\|^{2} & =1 .
\end{aligned}
$$

From which it follows that

$$
\left|\cos \left(\theta_{i j}\right)\right| \leq \sup \left(\sup _{a^{\prime} \neq a, b} \frac{\left|J_{i, a^{\prime}} \cap J_{j, b}\right|}{\sqrt{\left|J_{i, a^{\prime}}\right|\left|J_{j, b}\right|}}, \sup _{b} \frac{1}{\sqrt{\left|J_{j, b}\right|}}\right) .
$$

This last quantity also tends to 0 at the large size limit, since the cardinality of fibers tends to infinity. Therefore the dynamics of $\mathcal{M}^{\prime}$ is dominated by $\mu_{2}$. 
Now if several fibers are pruned which do not intersect, the above argument still holds and one still has a (46)-like upper bound: the dynamics is dominated by the largest remaining eigenvalue. Thus we have demonstrated that in this latter case, the pruned fibers can be bypassed via the remaining fast fibers which connect the configurations under concern to the bulk of the configuration set. It is therefore quite natural to believe that the same result still holds if some pruned fibers intersect: if all configurations are still connected to the bulk by at least one fast fiber, then the dynamics will remain rapidly mixing. As a conclusion, pruning all slow fibers should restore a fast dynamics provided the configuration set remains connected after pruning.

However very rare events could happen where this fiber-pruning process isolates completely some configurations, in that sense that the 4 fibers these configurations belong to are slow and need to be pruned. In that case, we would only have established that the process is rapidly mixing on all but very few tilings. Note that such an hypothetical restriction would not prevent one from speaking of rapidly mixing process since it would be rapid on typical tilings.

\section{References}

[1] D. Shechtman, I. Blech, D. Gratias, J.W. Cahn, Metallic phase with long-range orientational order and no translational symmetry, Phys. Rev. Lett. 53, 1951 (1984).

[2] R. Penrose, The role of aesthetics in pure and applied mathematical research, Bull. Inst. Math. Appl. 10, 226 (1974).

[3] D. Levine, P.J. Steinhardt, Quasicrystals: a new class of ordered structure, Phys. Rev. Lett. 53, 2477 (1984).

[4] V. Elser, Comment on "Quasicrystals: a new class of ordered structure", Phys. Rev. Lett. 54, 1730 (1985).

[5] H. Cohn, M. Larsen, J. Propp, The shape of a typical boxed plane partition, New York J. of Math. 4, 137 (1998).

[6] M. Latapy, Generalized integer partitions, tilings of zonotopes and lattices, to appear in Proceedings of the 12-th International Conference on Formal Power Series and Algebraic Combinatorics (Springer).

[7] C.L. Henley, in Quasicrystals, the state of the art, Ed. D.P. Di Vincenzo, P.J. Steinhardt, 429 (World Scientific, 1991).

[8] S. Lyonnard, G. Coddens, Y. Calvayrac, D. Gratias, Atomic (phason) hopping in perfect icosahedral quasicrystals $\mathrm{Al}_{70.3} \mathrm{Pd}_{21.4} \mathrm{Mn}_{8.3}$ by time-of-flight quasielastic neutron scattering, Phys. Rev. B 53, 3150 (1996).

[9] P.A. Kalugin, A. Katz, A mechanism for self-diffusion in quasi-crystals, Europhys. Lett. 21 (9), 921 (1993).

[10] R. Blüher, P. Scharwaechter, W. Frank, H. Kronmüller, First low-temperature radiotracer studies of diffusion in icosahedral quasicrystals, Phys. Rev. Lett. 80, 1014 (1998). 
[11] D. Caillard, Dislocations in quasicrystals, in Quasicrystals, current topics (World Scientific, Singapore, 2000).

[12] L.H. Tang, Random-tiling quasicrystal in three dimensions, Phys. Rev. Lett. 64, 2390 (1990).

[13] L.J. Shaw, V. Elser, C.L. Henley, Long-range order in a three-dimensional random-tiling quasicrystal, Phys. Rev. B. 43, 3423 (1991).

[14] M. Widom, N. Destainville, R. Mosseri, F. Bailly, Two-dimensional random tilings of large codimension, in Proceedings of the 6th International Conference on Quasicrystals (World Scientific, Singapore, 1998).

[15] D. Randall and P. Tetali, Analyzing Glauber dynamics by comparison of Markov chains, J. Math. Phys. 41 (3), 1598 (2000).

[16] M. Luby, D. Randall, A. Sinclair, Markov chain algorithms for planar lattice structures, to appear in SIAM Journal on Computing.

[17] D.B. Wilson, Mixing times of lozenge tiling and card shuffling Markov chains, preprint (arXiv:math.PR/0102193).

[18] C.L. Henley, Relaxation time for a dimer covering with height representation, J. Stat. Phys. 89, 483 (1997).

[19] N. Destainville, R. Mosseri, F. Bailly, Fixed-boundary octagonal random tilings: a combinatorial approach, J. Stat. Phys. 102, Nos 1/2, 147 (2001).

[20] N. Destainville, Entropy and boundary conditions in random rhombus tilings, J. Phys. A: Math. Gen. 31, 6123 (1998).

[21] N. Destainville, Flip dynamics in octagonal tiling sets, preprint, submitted to Phys. Rev. Lett. (arXiv:cond-mat/0101413).

[22] R. Mosseri, F. Bailly, Configurational entropy in octagonal tiling models, Int. J. Mod. Phys. B, Vol 7, 6\&7, 1427 (1993).

[23] A.J. Sinclair, Algorithms for random generation and counting: a Markov chain approach (Birkhäuser, Boston, 1993).

[24] G.R. Grimmett, D.R. Stirzaker, Probability and random processes, (Oxford University Press, 1982).

[25] R. Kenyon, Tiling a Polygon with parallelograms, Algorithmica 9, 382 (1993).

[26] D. Aldous, Random walks on finite groups and rapidly mixing Markov chains, Séminaire de probabilités XVII, Springer Lecture Notes in Mathematics 986, 243 (1981/1982).

[27] N.G. de Bruijn, Algebraic theory of Penrose's non-periodic tilings of the plane, Kon. Nederl. Akad. Wetensch. Proc. Ser. A 43, 84 (1981).

[28] N.G. de Bruijn, Dualization of multigrids, J. Phys. France 47, C3-9 (1986). 
[29] P. Diaconis, D. Strook, Geometric bounds for eigenvalues of Markov chains, The Annals of Applied Probability 1, 36 (1991).

[30] E.J. Gumbel, Statistics of extreme (Columbia, Columbia University Press, 1958).

[31] J.-P. Bouchaud, M. Mézard, Universality class for extreme-value statistics, J. Phys. A: Math. Gen. 30, 7997 (1997).

[32] R. Mosseri, J.-F. Sadoc, Glass-like properties in quasicrystals, Proceedings of the 5th International Conference on Quasicrystals, Ed. C. Janot, R. Mosseri (World Scientific, 1995), p. 747.

[33] L. Leuzzi, G. Parisi, Thermodynamics of a tiling model, J. Phys. A: Math. Gen 33, 4215 (2000).

[34] F. Gähler, J. Rhyner, Equivalence of the generalized grid and projection methods for the construction of quasiperiodic tilings, J. Phys. A: Math. Gen. 19, 267 (1986).

[35] P. Diaconis, L. Saloff-Coste, Comparison theorems for reversible Markov chains, Annals of Applied Probability 3, 696 (1993).

[36] J.-P. Bouchaud, A. Georges, Anomalous diffusion in disordered media: statistical mechanisms, models and physical applications, Phys. Rep. 195, 127 (1990). 